\title{
The Effect of Sandblasting of UHMWPE Substrate on the Wear Resistance of Hydrogen-Containing Carbon Coating
}

\author{
Yoshinori Takeichi ${ }^{1)^{*}}$, Takafumi Higashiyama ${ }^{1)}$, Takahiro Nakahigashi ${ }^{2)}$, \\ Yoshikazu Tanaka ${ }^{2)}$, Masanori Tsujioka ${ }^{2)}$ and Masao Uemura' ${ }^{1)}$ \\ ${ }^{1)}$ Department of Mechanical Engineering, Toyohashi University of Technology \\ 1-1, Hibarigaoka, Tempaku-cho, Toyohashi, Aichi 441-8580, Japan \\ ${ }^{2)}$ Nippon ITF Inc. \\ 575 Kuze Tonoshiro-cho, Minami-ku, Kyoto 601-8205, Japan \\ *Corresponding author: takeichi@mech.tut.ac.jp \\ ( Manuscript received 4 January 2008; accepted 14 March 2008; published 15 April 2008 ) \\ ( Presented at JAST Tribology Conference Saga, September 2007)
}

\begin{abstract}
Surface asperities were made on the surface of UHMWPE by sandblasting with sodium hydrogen carbonate abrasive in order to enhance the adhesion of hydrogen-containing carbon (HCC) coating to substrate. Sodium hydrogen carbonate abrasive was used because it easily solutes in hot water and the blasted abrasive stuck into the surface of UHMWPE can be entirely removed by washing with hot water. The HCC coating was deposited on the polished UHMWPE substrate and blasted substrate by RF-plasma CVD method. Friction test was conducted with the ball-on-disk tribometer in the distilled water. As the results of SEM observation and Raman spectroscopy analysis of the friction track on the HCC coating, it was confirmed that a substantial quantity of HCC coating deposited on the blasted substrate was remained on the friction track as compared with the polished substrate. It is concluded that the appropriate blasting treatment of UHMWPE substrate enhances the adhesiveness of the HCC coating to the substrate and improves wear resistance of HCC coating.
\end{abstract}

Keywords: hydrogen-containing carbon coating, UHMWPE, adhesion, surface asperity, sandblasting, sodium hydrogen carbonate abrasive

\section{Introduction}

Diamond-like carbon (DLC) coating is applied to many kinds of machine tools or devices because of its low friction coefficient, high wear resistance and chemical inertness. Recently DLC coating is applied to some mechanical parts of automobiles ${ }^{1)}$. Although DLC coating is practically used in a lot of applications, the substrates on which a DLC coating can be deposited has been limited to hard materials like metal or ceramics because of the large internal stress of DLC coating. On the other hand, there are requirements for forming tribo-coating on easily deformable substrate such as polymer materials. Nakahigashi et al. have developed a novel coating method to deposit "Flexible-DLC" coating on rubber or resin ${ }^{2)}$. This is the hydrogen-containing carbon (HCC) coating. Although $\mathrm{HCC}$ coating is formed in a similar process to that of forming DLC coating on metal or ceramics, the mechanical properties of HCC coating is different from that of DLC coating. This coating is practically applied to a rubber O-ring seal in a zoom lens of camera to reduce the friction resistance between O-ring and lens barrel without using grease.

One of the biotribological issues is the short duration of artificial hip joint. Various efforts are being made to reduce the wear of ultra-high molecular weight polyethylene (UHMWPE) used in the artificial joint. It is reported that the generation of the cross-links of polyethylene molecules by gamma-ray irradiation improves wear resistance of $\mathrm{UHMWPE}^{3)}$. On the contrary, it is reported that the irradiation of gamma-ray and ageing increase abrasive wear of UHMWPE ${ }^{4,5)}$. Coating also is the promising approach to reduce the wear of UHMWPE. It was reported that DLC coating deposited on the surface of metal or ceramic femoral head of artificial joint reduced the wear of UHMWPE acetabular $\operatorname{cup}^{6-8)}$. Recently the biocompatibilities of DLC coating were confirmed ${ }^{8,9}$.

It is supposed that depositing tribo-coating not only on the femoral head but also on the UHMWPE acetabular cup could improve the wear of artificial joint. Since UHMWPE shows low friction coefficient (0.1-0.2) when it slides against metal surface, it isn't expected that the coating on the UHMWPE reduces friction coefficient in comparison with the friction coefficient of uncoated UHMWPE. It is, however, expected that the coating reduces the wear of UHMWPE. Thus we have tried forming HCC coating on the UHMWPE substrate and the tribological properties of this coating were studied ${ }^{10)}$. As the results, 
a further improvement of the adhesion of the HCC coating to the UHMWPE substrate became an important issue.

Improvement of adhesion of DLC coating to the hard substrate has been attained by various methods such as interlayer forming, multilayer forming, graded composition film forming or other material doping ${ }^{11-13)}$. However, these methods are not applicable for the artificial hip joint because the materials contained in the coating are restricted due to the biocompatibilities. On the other hand, making appropriate asperities on the surface of substrates is one of the conventional methods to improve the adhesion of coating. It was reported that the adhesion of DLC coating on metal substrate was improved by roughen the surface of substrate ${ }^{14)}$. In this experiment, the surface of UHMWPE substrate was abraded by sandblasting method in order to enhance the adhesion of HCC coating to substrate and then improve the wear resistance of coating.

\section{Samples and experiments}

\subsection{Preparation of substrates}

Alumina, silicon carbide and metal powder are widely used as a sandblasting abrasive. The hard abrasive easily stuck into the soft UHMWPE surface and it is difficult to remove the stuck abrasive. Therefore sodium hydrogen carbonate $\left(\mathrm{NaHCO}_{3}\right)$ powder was used as a sandblasting abrasive because it is soluble in hot water and could be easily removed by washing in hot water. Sodium hydrogen carbonate is a commonly-used material which is used for cooking as a leavening agent (baking powder), for cleaning as an abrasive powder and for medical as an antacid to treat acid indigestion and pyrosis. The alumina $\left(\mathrm{Al}_{2} \mathrm{O}_{3}\right)$ powder, which is the conventional sandblasting abrasive, was used in this experiment for comparison. Fig. 1(a) and 1(b) show the SEM (Scanning electron microscopy) images obtained from the sodium hydrogen carbonate abrasive with a nominal average particle size of 105 $149 \mu \mathrm{m}$ and the aluminum abrasive with that of $88-125$ $\mu \mathrm{m}$, respectively. Sodium hydrogen carbonate abrasive is columnar grain with waney edge. Alumina abrasive is angular grain with steep edge.

The medical grade UHMWPE (Chirulen, GUR412) was used as a substrate of the HCC coating. The circular disk substrate with the diameter of $30 \mathrm{~mm}$ and the thickness of $3 \mathrm{~mm}$ was machined from a UHMWPE rod. First the surface of all UHMWPE disk was polished by emery paper (\#2000) with pouring water and then half of them were blasted by the sandblasting machine (Nicchu, Petit Blast PDM-03B). The blasting period was varied from 1 to 15 minutes. The compressor pressure and the blasting distance between the nozzle and disk were controlled to be constant. After the sandblasting, substrates were washed with hot water $\left(\sim 50^{\circ} \mathrm{C}\right)$ and then cleaned ultrasonically with distilled water.
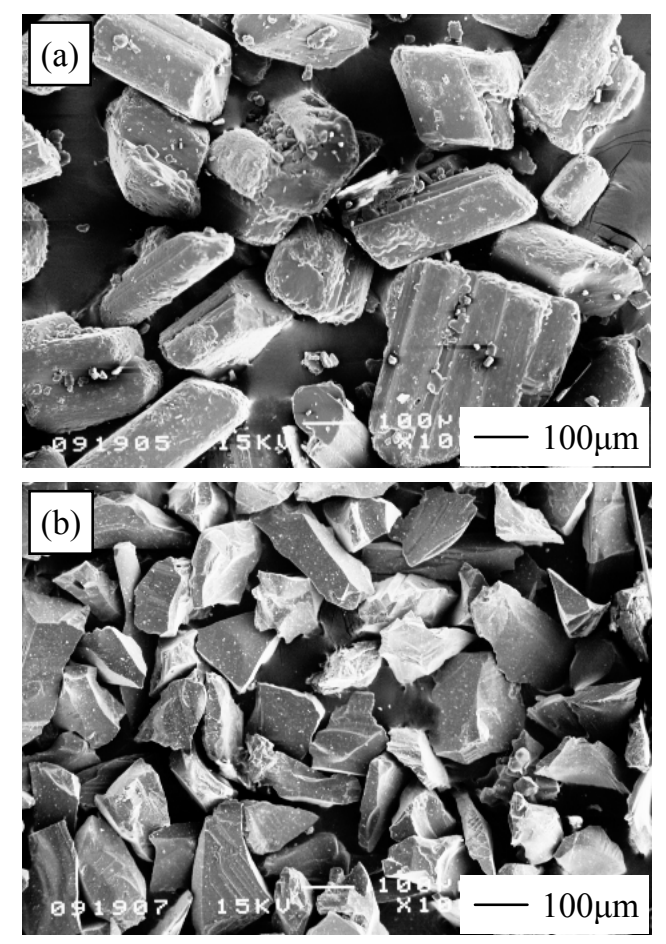

Fig. 1 SEM images of (a) sodium hydrogen carbonate abrasive and (b) alumina abrasive.

\subsection{HCC coating}

The HCC coating was deposited on the polished UHMWPE substrate and on the blasted substrate by the amplitude-modulated radio frequency plasma chemical vapor deposition (RF P-CVD) method. Methane $\left(\mathrm{CH}_{4}\right)$ was used as a processing gas. The UHMWPE disk was placed on the high frequency electrode which was cooled with water. The temperature of sample was kept to be less than $60{ }^{\circ} \mathrm{C}$ during the deposition process. At first, the surface of UHMWPE was cleaned with $\mathrm{H}_{2}$ plasma for the purpose of removing surface contamination and then HCC coating was deposited. The thickness of the HCC coating was about $1 \mu \mathrm{m}$ and its hardness was about $100 \mathrm{HV}$. The coating contains 20 - $40 \%$ of hydrogen.

\subsection{Friction test}

Ball-on-disk friction tests were carried out. Stainless steel ball (SUS316) with a diameter of $6 \mathrm{~mm}$ was used as a ball slider and HCC coated UHMWPE disk was used as a disk specimen. The ball specimen was ultrasonically cleaned with acetone and with distilled water before the friction test. The disk specimen was washed with mild detergent and then ultrasonically cleaned with distilled water before the friction test. The normal load was $1.96 \mathrm{~N}$ and the sliding speed was controlled to be $22 \mathrm{~mm} / \mathrm{sec}$. The total sliding cycle was 432,000 revolutions, and this corresponds to the sliding period of 24 hours and the sliding distance of $19 \mathrm{~km}$. Friction tests were performed in the distilled water as lubricant and its temperature was controlled to be about $37^{\circ} \mathrm{C}$. 


\section{Results and discussion}

\subsection{Observation of samples}

SEM images of the surface of UHMWPE polished with emery paper and that blasted with alumina abrasive are shown in Fig. 2(a) and 2(b), respectively. SEM images of the surface of UHMWPE substrate blasted with sodium hydrogen carbonate abrasive for 1,5 and $15 \mathrm{~min}$ are shown in Fig. 2(c), 2(d) and 2(e), respectively. All images are obtained after washed in hot water and ultrasonically cleaned with distilled water. The images of blasted surface (Fig. 2(b) - 2(e)) show different surface topography in comparison with the polished surface (Fig. 2(a)). The surface of UHMWPE
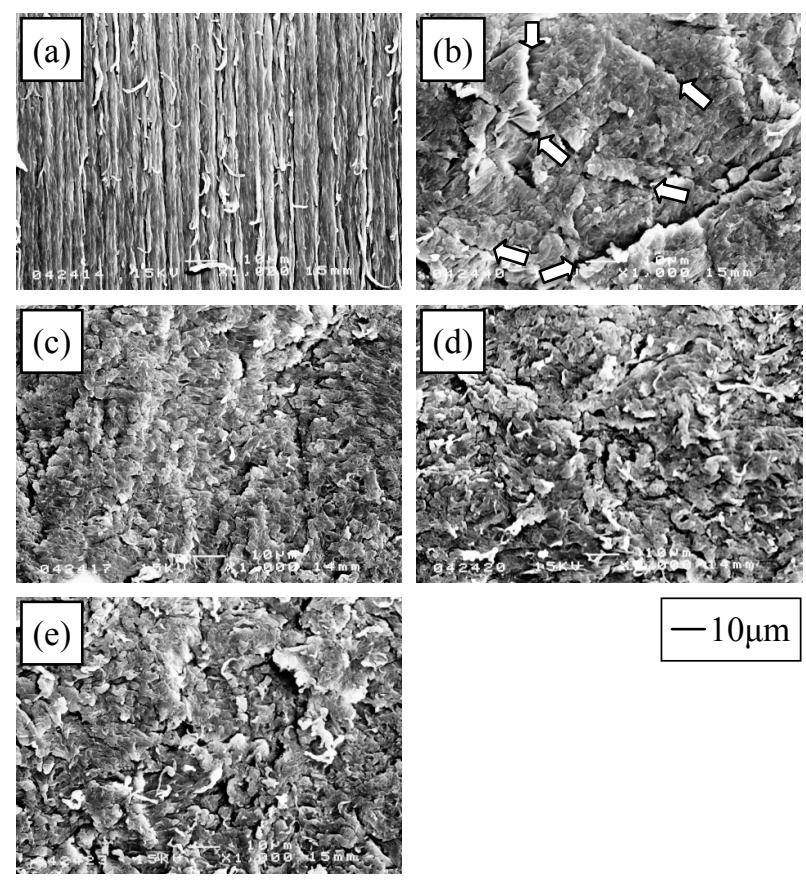

Fig. 2 SEM images of the surface of UHMWPE (a) polished with emery paper, (b) blasted with alumina abrasive and blasted with sodium hydrogen carbonate abrasive for (c) $1 \mathrm{~min}$, (d) 5 min and (e) 15 min.
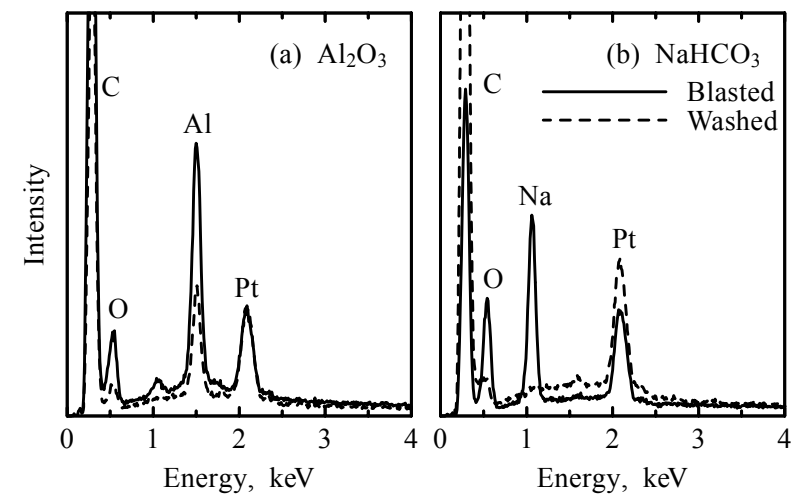

Fig. 3 EPMA spectra obtained from the surface of UHMWPE blasted with (a) the alumina and (b) the sodium hydrogen carbonate abrasives before and after the washing treatment. blasted with alumina abrasive seems to be chopped roughly with a sharp blade. There are many long grooves as indicated with arrow in Fig. 2(b). On the other hand, as shown in Fig. 2(c) - 2(e), the surface blasted with sodium hydrogen carbonate abrasive seems to be finely abraded as a whole. The polishing direction is from top to bottom in every image. The polished direction is distinguishable in Fig. 2(a). On the other hand, the blasted surfaces show randomly abraded surface and the polished direction is undistinguishable as shown in Fig. 2(b) - 2(e). The surface blasted with sodium hydrogen carbonate abrasive for 5 and $15 \mathrm{~min}$ show high asperity in comparison with that for $1 \mathrm{~min}$. The change of the surface topography seems to be saturated in $5 \mathrm{~min}$ of blasting period.

EPMA (Electron probe microanalysis) spectra obtained from the surface of UHMWPE blasted with alumina abrasive and sodium hydrogen carbonate abrasive are shown in Fig. 3(a) and 3(b), respectively. The spectra obtained before and after the washing treatment are shown in each figure. Platinum was detected from both surfaces because the surface of UHMWPE was coated with platinum to get electric conductivity and to suppress electric charged up. Thus the peak of platinum can be neglected. In the EPMA spectra obtained from the surface blasted with alumina, though the peak of aluminum becomes small after the washing treatment, it still remains with appreciable intensity. Therefore it can be considered that small particles of alumina abrasive buried into the surface remain after the washing treatment. On the other hand, in the spectra obtained from the surface blasted with sodium hydrogen carbonate, the peak of sodium is disappeared after the washing treatment. It shows that the particles of sodium hydrogen carbonate abrasive are completely removed from the surface of UHMWPE.

Fig. 4(a) - 4(d) show the SEM images of the surfaces of HCC coating deposited on the polished surface and blasted surface for 1, 5 and 15 min. The surface of HCC coating consists of fused grains which have caterpillar like configuration. The orientation of these fused grains depends on the surface topography of its substrate. The polished direction is distinguishable even after the deposition of HCC coating as shown in Fig. 4(a). Although the topography of $1 \mathrm{~min}$ blasted substrate before coating (Fig. 2(c)) is definitely different from that of 5 and 15 min blasted substrate (Fig. 2(d) and 2(e)), a clear difference is not seen among the surfaces of HCC coating on the blasted substrate.

\subsection{Friction test and analysis}

The results of ball-on-disk friction test are shown in Fig. 5. All specimens show similar friction coefficient of around $0.17-0.18$ after 3 hours of sliding. The polished substrate and blasted substrates show different variation of friction coefficient for 3 hours of the beginning of sliding. The friction coefficient of polished substrate increased to 0.21 and then decreased to 0.17 during 3 

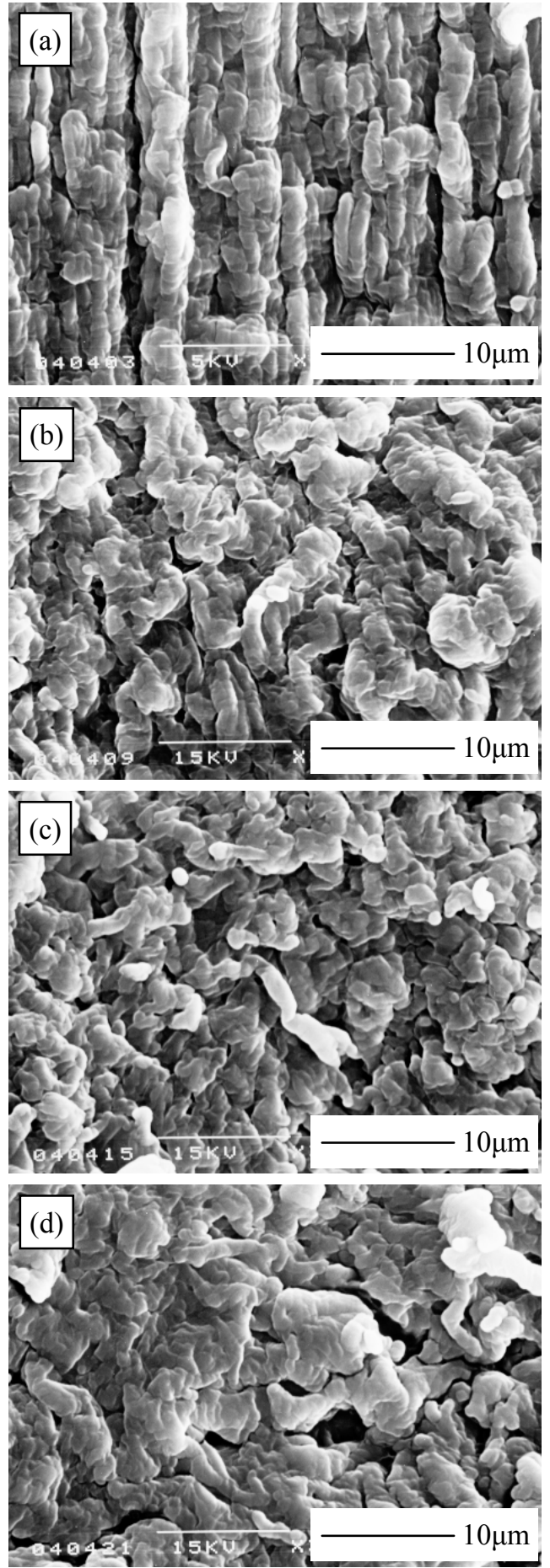

Fig. 4 SEM images of the surfaces of HCC coating deposited on (a) the polished substrate and (b) $/(\mathrm{c}) /(\mathrm{d})$ the substrate blasted for $1 / 5 / 15 \mathrm{~min}$.

hours of the beginning. This variation of friction coefficient at the beginning of sliding could be due to the low adhesion of HCC coating for the polished substrate. The friction coefficient will not increase drastically even if the HCC coating is entirely removed from the friction track, because UHMWPE shows low friction coefficient. Therefore it is difficult to say

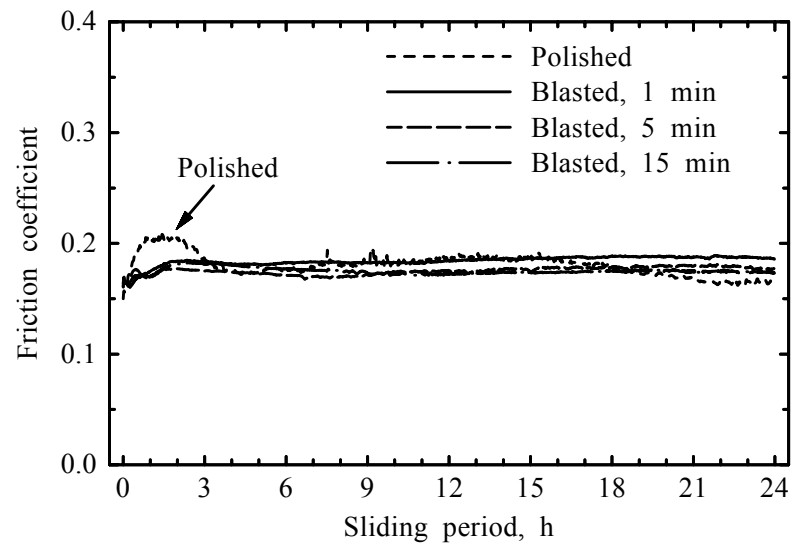

Fig. 5 Friction coefficient between the stainless steel ball and the HCC coating deposited on the polished and blasted UHMWPE substrates.

whether the HCC coating on the friction track remains or not, from the variation in friction coefficient.

SEM images of the friction scar of ball specimens are shown in Fig. 6(a) - 6(d). Sliding direction is from top to bottom in each picture. Large amount of wear debris are adhered on the ball specimen which rubbed HCC coating deposited on the polished substrate as shown in Fig. 6(a). As for the ball specimens which rubbed HCC coating deposited on the substrates blasted for 1 and $5 \mathrm{~min}$, small quantity of wear debris are adhered on the ball specimens as shown in Fig. 6(b) and 6(c). On the other hand, wear debris is not observed from the ball specimen which rubbed HCC coating deposited on the substrate blasted for $15 \mathrm{~min}$ as shown in Fig. 6(d). The EPMA mappings for carbon are shown in Fig. 7(a) - 7(d). Each mapping is obtained from the same area of the SEM image in Fig. 6(a) - 6(d). The brightness in the mapping expresses the quantity of detected carbon. The distributions of carbon are exactly same with that of adhered material on the ball specimens. No signal of carbon is detected from the friction scar of ball specimen which rubbed the specimen blasted for $15 \mathrm{~min}$.

Fig. 8(a) shows the SEM image of the friction track of the HCC coating deposited on the UHMWPE substrate blasted for $15 \mathrm{~min}$. Because of the asperity of substrate, several small concave areas are not rubbed during the friction test. It is difficult to confirm whether HCC coating was entirely removed away from the black colored friction scar in Fig. 8(a). Fig. 8(b) is the close-up image of a boundary of friction track. From this image, it can be confirmed that the caterpillar like fused grains of HCC coating is flatten out and becomes smooth in the friction track. It is conceivable that the HCC coating remains to some extent on the friction track. Unfortunately, the thickness of remaining HCC can not be evaluated by the conventional qualitative analysis such as EPMA or Auger Electron Spectroscopy because these methods can not distinguish the carbon of HCC from the carbon of UHMWPE. Therefore friction 

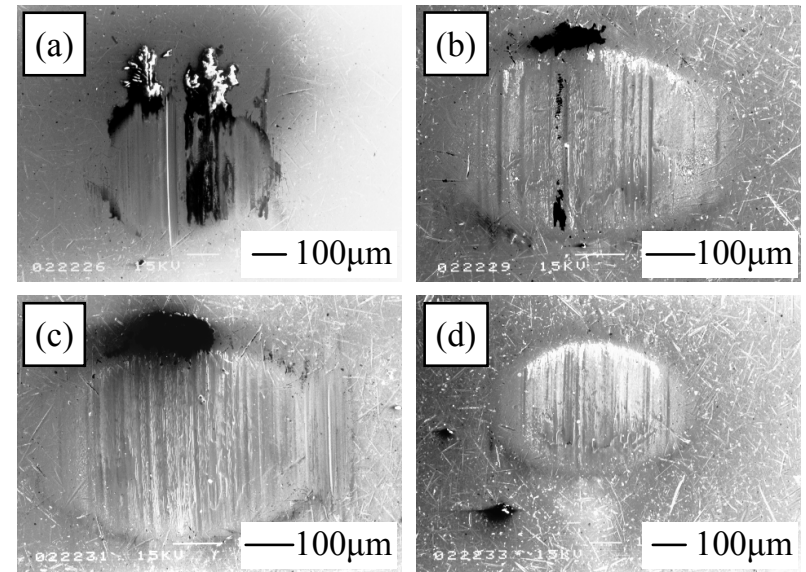

Fig. 6 SEM images of the friction scar on the ball specimens which rubbed HCC coating on (a) the polished substrate and $(\mathrm{b}) /(\mathrm{c}) /(\mathrm{d})$ the substrate blasted for $1 / 5 / 15 \mathrm{~min}$.
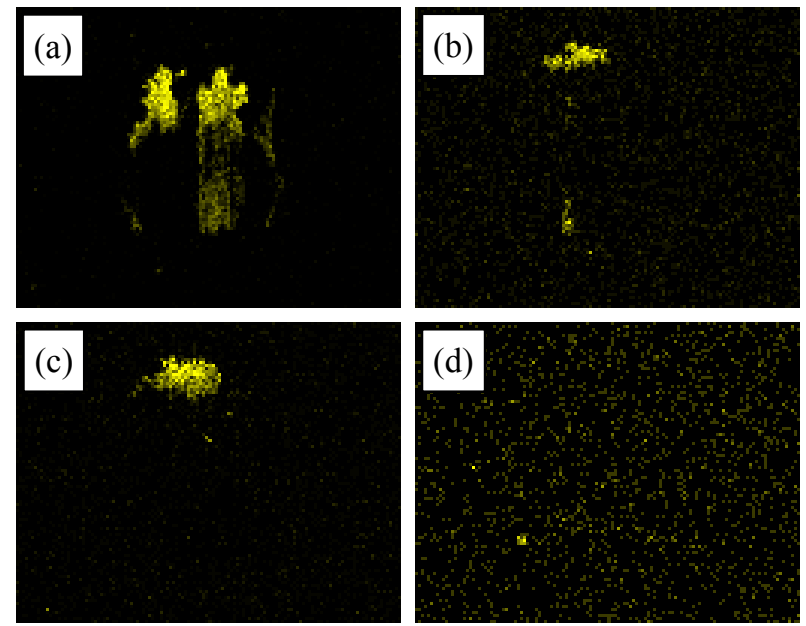

Fig. 7 EPMA mappings for carbon on the ball specimens which rubbed HCC coating.

track of HCC coating was analyzed by Raman spectroscopy.

Fig. 9 shows the Raman spectra obtained from the UHMWPE substrate, the HCC coating deposited on the UHMWPE substrate and the HCC powder scraped off from the HCC coating deposited on UHMWPE. For comparison, the Raman spectrum obtained from the DLC coating deposited on the steel substrate is shown in Fig. 9. UHMWPE shows sharp twin peaks at around $2800-2900 \mathrm{~cm}^{-1}$. On the other hand, the HCC powder scraped off from the HCC coating shows large background of spectrum and no sharp peak. The D-peak and G-peak shown in the spectrum of DLC coating are not observed from the HCC powder. The HCC coating deposited on the UHMWPE shows the spectrum which is the superposition of the spectrum of UHMWPE substrate and the spectrum of the HCC powder because the signal is obtained not only from the HCC coating but also from the UHMWPE substrate. Therefore the sharp peaks at around $2800-2900 \mathrm{~cm}^{-1}$ increase and the
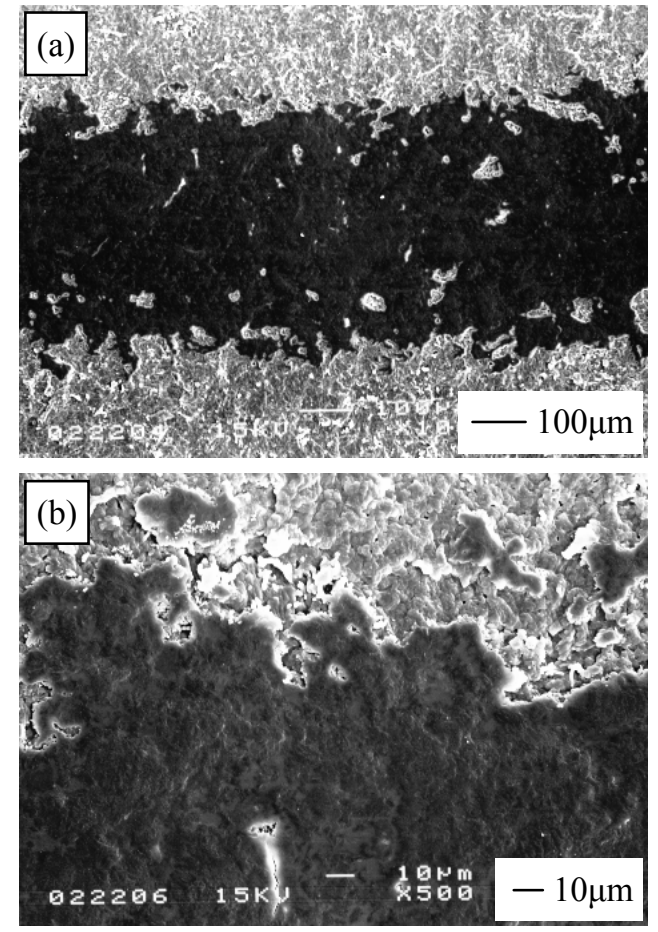

Fig. 8 SEM images of (a) the friction track on the HCC coating and (b) the close-up image of a boundary of the friction track.

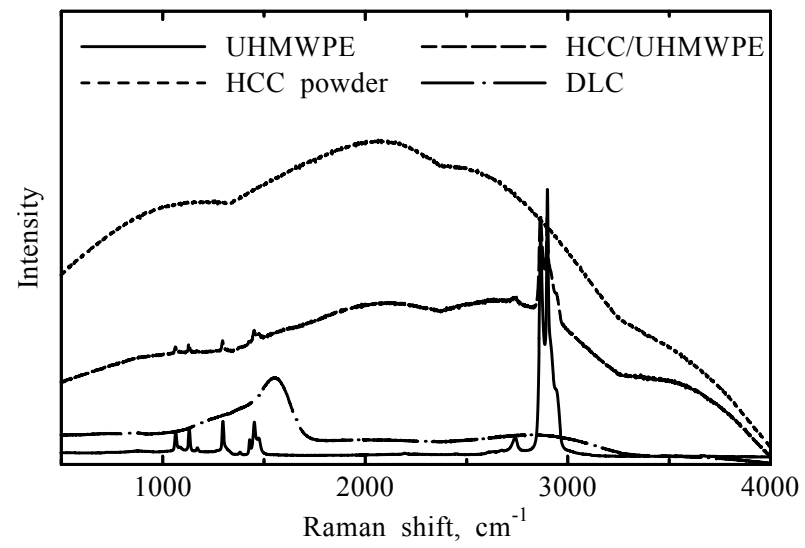

Fig. 9 Raman spectra obtained from various sample.

background decreases with decrease in the thickness of HCC coating.

Fig. 10(a) and 10(b) show Raman spectra obtained from the friction track of the HCC coating deposited on the polished and blasted (15 min) substrate, respectively. Spectra were obtained before the friction test and after 6 , 12 and 24 hours of sliding periods. As for the polished substrate, the background of the spectrum becomes small with the sliding period. The large background of the spectrum can not be observed after 12 hours of sliding period and the shape of these spectra are same with that obtained from UHMWPE. On the contrary, as for the blasted substrate, although the background becomes small with the sliding period, a certain level of background still remains in the spectrum after 24 hours 

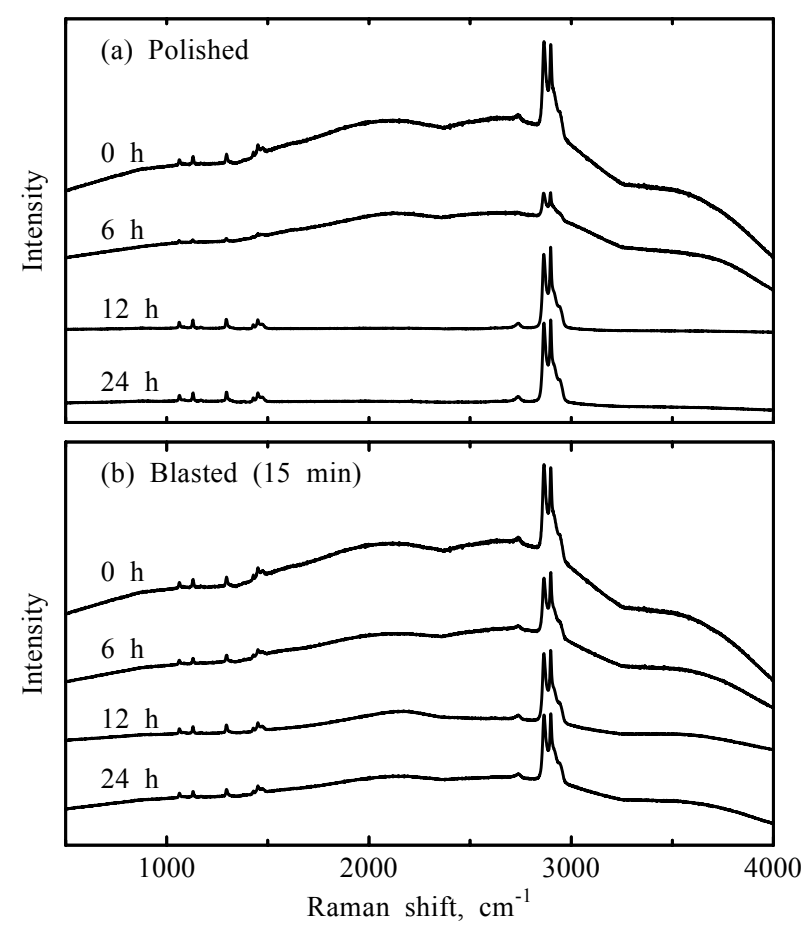

Fig. 10 Raman spectra obtained from the HCC coating on the polished substrate and (b) the blasted substrate.

of sliding period. From these results, it is confirmed that the HCC coating remains on the UHMWPE substrate with substantial thickness after 24 hours of sliding period for the blasted substrate. Therefore it can be concluded that the appropriate blasting treatment of UHMWPE substrate and the complete removal of sandblasting abrasive could enhance the adhesion of the $\mathrm{HCC}$ coating and improve the wear resistance of $\mathrm{HCC}$ coating.

\section{Conclusions}

In order to enhance the adhesion of HCC coating to UHMWPE substrate, the surface of UHMWPE was blasted with the sodium hydrogen carbonate abrasive. The abrasive stuck into the surface of substrate are entirely removed by washing with hot water.

The HCC coating was deposited on the surface of polished or blasted substrate and the ball-on-disk friction test was conducted. As the results of SEM observation and Raman spectroscopy analysis of friction track, it is concluded that the appropriate blasting of the surface of UHMWPE substrate with sodium hydrogen carbonate abrasive enhances the adhesion of HCC coating to the polymer substrate and improves the wear resistance of HCC coating on the UHMWPE substrate.

\section{References}

[1] Kano, M., "Super Low Friction of DLC Applied to Engine Cam Follower Lubricated with
Ester-Containing Oil," Tribology International, 39, 12, 2006, 1682-1685.

[2] Nakahigashi, T., Tanaka, Y., Miyake, K. and Oohara, H., "Properties of Flexible DLC Film Deposited by Amplitude-Modulated RF P-CVD," Tribology International, 37, 2004, 907-912.

[3] Oonishi, H., Takayama, Y. and Tsuji, E., "Improvement of Polyethylene by Irradiation in Artificial Joints," Radiation Physics and Chemistry, 39, 6, 1992, 495-504.

[4] Choudhury, M. and Hutchings, I. M., "The Effects of Irradiation and Ageing on the Abrasive Wear Resistance of Ultra High Molecular Weight Polyethylene," Wear, 203-204, 1997, 335-340.

[5] Lee, K. Y. and Lee, K. H., "Wear of Shelf-Aged UHMWPE Acetabular Liners," Wear, 225-229, 1999, 728-733.

[6] Anttila, A., Lappalainen, R., Heinonen, H., Santavirta, S. and Konttinen, Y. T., "Superiority of Diamondlike Carbon Coating on Articulating Surfaces of Artificial Hip Joints," New Diamond and Frontier Carbon Tech., 9, 4, 1999, 283-288.

[7] Dowling, D. P., Kola, P. V., Donnelly, K., Kelly, T. C., Brumitt, K., Lloyd, L., Eloy, R., Therin, M. and Weill, N., "Evaluation of Diamono-Like Carbon-Coated Orthopaedic Implants," Diamond and Related Materials, 6, 1997, 390-393.

[8] Grill, A., "Diamond-Like Carbon Coatings as Biocompatible Materials -An Overview," Diamond and Related Materials, 12, 2003, 166-170.

[9] Hauert, R., Müller, U., Francz, G., Birchler, F., Schroeder, A., Mayer, J. and Wintermantel, E., "Surface Analysis and Bioreactions of $\mathrm{F}$ and $\mathrm{Si}$ Containing a-C:H," Thin Solid Films, 308-309, 1997, 191-194.

[10] Takeichi, Y., Nakahigashi, T., Tanaka, Y., Yamane, T. and Uemura, M., Proc. of the 4th International Biotribology Forum \& the 24th Biotribology Symposium, Fukuoka, 2003, 33-36.

[11] Klaffke, D., Santner, E., Spaltmann, D. and Woydt, M., "Influences on the Tribological Behaviour of Slip-Rolling DLC-Coatings," Wear, 259, 1-6, 2005, 752-758.

[12] Zhang, W. and Tanaka, A., "Tribological Properties of DLC Films Deposited under Various Conditions Using a Plasma-Enhanced CVD," Tribology International, 37, 11-12, 2004, 975-982.

[13] Miyoshi, K., Pohlchuck, B., Street, K. W., Zabinski, J. S., Sanders, J. H., Voevodin, A. A. and Wu, R. L. C., "Sliding Wear and Fretting Wear of Diamondlike Carbon-Based, Functionally Graded Nanocomposite Coatings," Wear, 225-229, 1, 1999, 65-73.

[14] Kameyama, Y. and Komotori, J., "Tribological Properties of Structural Steel Modified by Fine Particle Bombardment (FPB) and Diamond-Like Carbon Hybrid Surface Treatment," Wear, 263, 7-12, 2007, 1354-1363. 